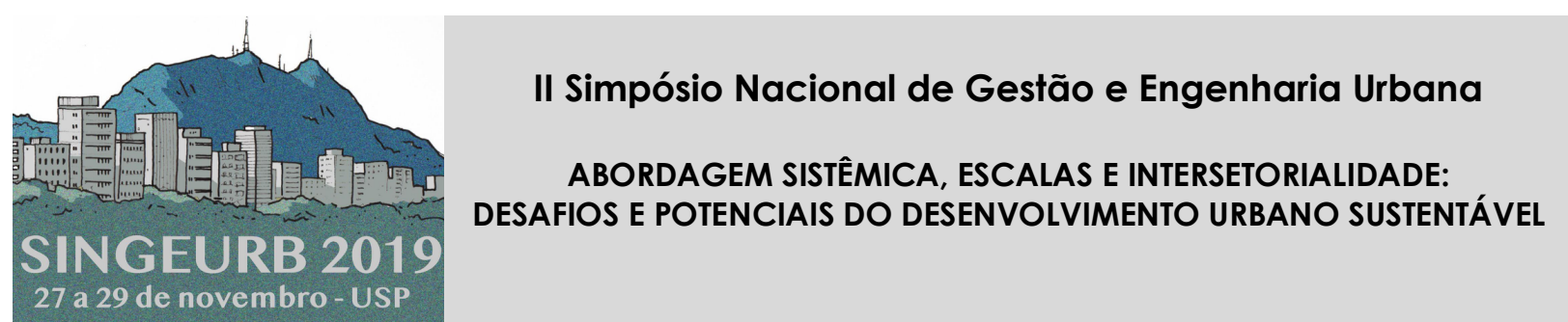

\title{
Geovista - Gestão descentralizada das condições do pavimento em ambiente urbano. ${ }^{1}$
}

\section{Geovista - Decentralized management of pavement conditions in an urban environment}

\author{
Paixão, M. J. S. '; Maranhão, F. L.2; \\ 1 Universidade de São Paulo (USP), Rua Paim, 296, apt.1604, São Paulo-SP, \\ Brasil, marciopaixao@usp.br \\ 2 Universidade de São Paulo (USP), flavio.maranhao@usp.br
}

\begin{abstract}
RESUMO
A principal problemática abordada nesta pesquisa discorre sobre a possibilidade de desenvolver, através da tecnologia disponível, um método mais rápido, simples e eficaz para gerir a qualidade do pavimento no ambiente urbano. Esta pesquisa está sendo desenvolvida de maneira multidisciplinar, envolvendo as áreas de engenharia de hardware, software, desenvolvimento de aplicativo para celular e engenharia civil. Cada área do conhecimento envolvida tem relevância fundamental para o atingimento das metas estabelecidas de entregar para a Prefeitura de Cidade de São Paulo um sistema que seja preciso, funcional, e com alto foco na gestão urbana, que por muitas vezes carece da informação mais relevante para as decisões e destinação de recursos. Consonante ao objetivo postulado o projeto está desenvolvendo um método simples de medida da condição do pavimento através de sensores sem fio instalados em veículos que mineram uma grande massa de dados, que são devidamente tratados para este fim. O projeto está em fase intermediária de desenvolvimento e os dados parciais estão doravante apresentados, de acordo com o processo estabelecido no âmbito da pesquisa.
\end{abstract}

Palavras-chave: Gestão Urbana, Pavimentos, Cidades Inteligentes.

\begin{abstract}
The main problem addressed in this research is the possibility of developing, through available technology, a faster, simpler and more efficient method to manage pavement quality in the urban environment. This research is being developed in a multidisciplinary way, involving the areas of hardware engineering, software, mobile application development and civil engineering. Each area of knowledge involved is fundamentally relevant to the achievement of the established goals of delivering to the City of São Paulo a system that is precise, functional and with a high focus on urban management, which often lacks the most relevant information for the decisions and allocation of resources. Consonant to the postulated objective the project is developing a simple method of measuring the condition of the pavement through wireless sensors installed in vehicles that mine a large mass of data,

1 PAIXÃO, M. J. S.; MARANHÃO, F. L. Geovista - Gestão descentralizada das condições do pavimento em ambiente urbano. In: II SIMPÓSIO NACIONAL DE GESTÃO E ENGENHARIA URBANA: SINGEURB, 2019, São Paulo. Anais... Porto Alegre: ANTAC, 2019.
\end{abstract}


which are properly treated for this purpose. The project is in the intermediate phase of development and the partial data are now presented, according to the process established in the scope of the research.

Keywords: Urban Management, Pavements, Smartcities.

\section{INTRODUÇÃO}

O cenário da administração pública das cidades brasileiras hoje se revela muito distante da inovação quando o assunto é gestão dos seus ativos. O que se dispõe hoje de tecnologia e inovação em modelos de Tecnologias de Informação e Comunicação (TICs) não é aproveitado a contento pelos seus administradores. Ademais, é percebido um distanciamento ainda maior quando tratamos da introdução de conceitos mais abrangentes como o City Information Modeling (CIM), Smartcities, Geografic Information System (GIS), entre outros conceitos que são tendência para o futuro da modelagem urbana (CTC 1).

Em se tratando de ativos públicos, a gestão, monitoramento, reparos e manutenção da qualidade do pavimento asfáltico das cidades sempre será um assunto de absoluta relevância. Na cidade de São Paulo, por exemplo, existem mais de $18.000 \mathrm{Km}$ de vias pavimentadas, nas quais transitam diariamente mais de oito milhões de veículos, conforme informações da CET, além de investimentos de grande vulto para a prefeitura.

Todavia, apesar de tamanha importância, não está estabelecida uma visão de antecipação aos problemas das vias, prevenção, preservação e longevidade do pavimento. Sendo, inclusive, difícil de obter a rastreabilidade de relatórios de qualidade, durabilidade, histórico de manutenção, e capacidade de suporte ao longo do tempo.

Entrando nesse mérito da avaliação dos pavimentos e traçando um paralelo com as projeções de futuro da administração das cidades, existem diversos estudos sendo elaborados ao redor do mundo em fase de pesquisa e desenvolvimento.

Muito promissores são sistemas que busquem minerar e gerir grandes quantidades de dados. Ao passo que, existindo em grande volume, tais informações quando submetidas ao tratamento adequado passam a ter a capacidade de convergir a algo que se torne relevante e conclusivo integrando-se no rol de ferramentas utilizadas para o planejamento das cidades.

De acordo com diversas entidades e órgãos responsáveis por gestão do pavimento, o principal indicador da sua condição, é a sua rugosidade superficial, Ainda de acordo com o cálculo do PSI (Present Serviceability Index), objetivamente, $95 \%$ do índice de classificação depende apenas da sua rugosidade. Para determinação deste índice, existem diversos métodos, os quais se diferenciam entre si numa escala inversamente proporcional entre a precisão do indicador e conveniência da obtenção do mesmo de determinada via, como está ilustrado no esquema abaixo (Fig.1). 
Figura 1 - Relação entre métodos de obtenção da rugosidade

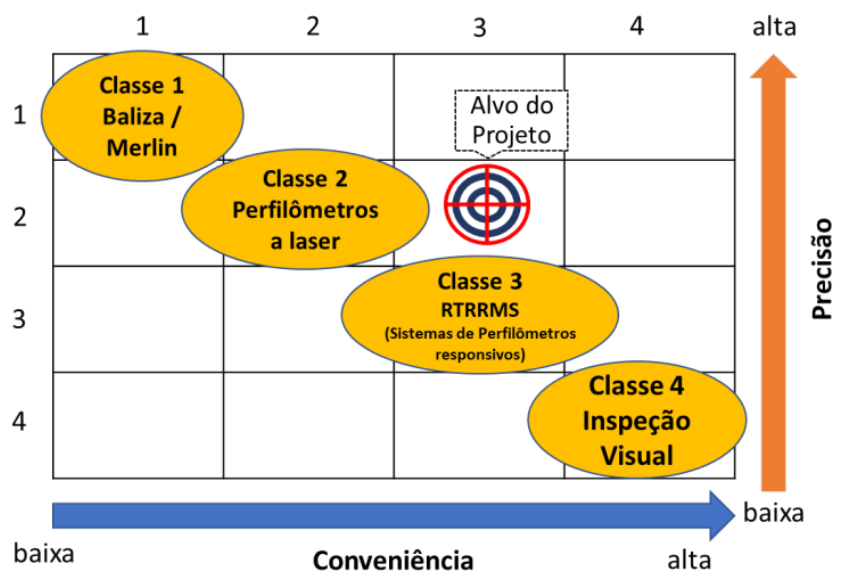

Fonte: Tomiyama (2017 - adaptado)

Entretanto, seguindo as tendências do futuro, podemos destacar a necessidade do desenvolvimento de um método inovador que contemple àquilo que os métodos tradicionais de avaliação do pavimento dispõem em termos de precisão, porém fazendo uso da capacidade tecnológica de resposta em tempo real dos grandes volumes de informação. A criação de um sistema que converge estes dois parâmetros é o que representa o cerne deste Projeto, endossado pela Prefeitura Municipal de São Paulo, que será apresentado neste artigo.

\section{METODOLOGIA}

O Projeto Geovista tem como objetivo suprir a Prefeitura de São Paulo de um sistema para avaliação da qualidade superficial do pavimento dos logradouros da cidade, que trabalhe em tempo real, de interface rápida e de fácil manuseio pela(s) equipe(s) pela gestão da Malha Viária da cidade.

Para tal, foi proposta a instalação de acelerômetros em uma frota de veículos que ao transitar pelas ruas, fazem o monitoramento descentralizado através das informações de rugosidade superficial. Com base nos resultados, as ruas serão classificadas pela sua condição e criados mapas atualizados, o que possibilitará a classificação da prioridade de intervenção em vias fruto do cruzamento dos dados obtidos, criticidade, e fluxo de veículos.

As ruas serão plotadas em mapas interativos com interface intuitiva e classificadas em duas camadas: em função do índice de irregularidade superficial, apresentada em cinco níveis (ótima, boa, regular, ruim e péssima) e, em outra camada, demonstrar-se-á as imperfeições pontuais como buracos, valetas, lombadas.

Para atingir os objetivos traçados, a estratégia adotada, resumidamente será:

- 1. Desenvolver Software de aquisição de dados de acelerômetros responsivos à vibração de rodas de veículos;

- 2. Desenvolver e instalar Hardware, com sensores e de aceleração, conjuntamente com uma solução de armazenamento provisório de dados e GPS para geolocalização;

- 3. Armazenar e transmitir um sistema central em a nuvem as informações de vibração geolocalizadas;

- 4. Processar as informações recebidas através de algoritmos com o objetivo da avaliação da rugosidade do pavimento e defeitos pontuais;

- 5. Criar visões em mapas eletrônicos que interessem à PMSP;

- 6. Atualizar constantemente os mapeamentos das condições das vias ao longo do tempo; 
- 7. Validar as informações de leituras e processamento obtidas através de pesquisa científica.

\subsection{Software para aquisição de dados - Data mining}

O aplicativo Android do Projeto, desenvolvido para a aquisição de dados, foi concebida voltada para smartphones de linha econômica, por ser uma tendência mundial de utilizar a base destas plataformas para o desenvolvimento de aplicações e facilitar a atualização remota dos softwares utilizados. Além do que, converge para o objetivo de tornar a aplicação barata, impulsionando a aquisição em massa de dados.

As informações coletadas pelo software são: Timestamp, latitude, longitude, quantidade de satélites, erros de localização, velocidade de deslocamento e aceleração da gravidade nos eixos $X, Y$ e Z. Nessas aquisições é utilizada a frequência de $10 \mathrm{~Hz}$ que significa 10 medições a cada segundo. A figura 2 abaixo corresponde à captura da tela do smartphone com o aplicativo em funcionamento.

Figura 2 - Capturas de tela do App do Geovista
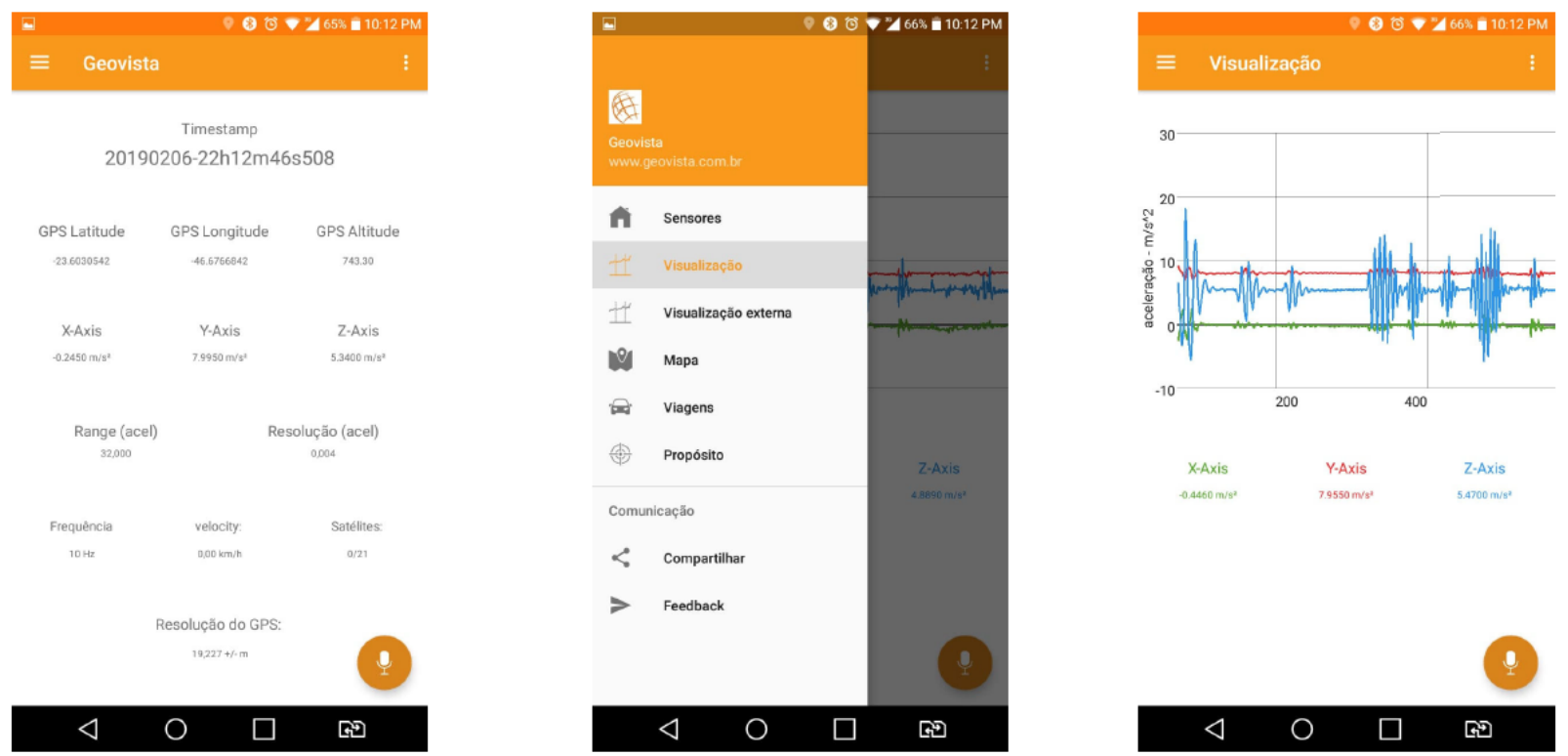

Fonte: Os autores (2019)

O aplicativo está configurado para concentrar-se no registro dos dados mesmo rodando em segundo plano e pode, inclusive, se conectar com outros sensores específicos instalados em outros pontos do carro. As informações coletadas geram arquivos que são armazenados provisoriamente no próprio smartphone e, têm um gatilho de envio ao servidor em nuvem a cada nova conexão com qualquer rede wi-fi encontrada. Tais ajustes ainda são experimentais e serão aperfeiçoados ao passo que estejam instalados na frota prevista e se necessite da massificação e periodicidade padronizada dos mesmos

\subsection{Modelagem e Implementação do banco de dados do sistema}

Para a implementação do projeto é necessário criar as bases de dados no modelo correto para que sejam armazenadas organizadamente todas as informações relevantes, bem como o relacionamento entre essas informações, tal planejamento denomina-se "modelagem de dados".

A modelagem de dados ocorre em duas esferas: (i) no ambiente dos dados que são cadastrados ou coletados quanto à rugosidade de cada trecho de logradouro e todos os relacionamentos necessários para a precisão dessa informação e (ii) no conceito de data 
warehouse de consolidação dessas informações e criação de visões pré-processadas em cubos multidimensionais para que, ao ser solicitados, os mapas e relatórios de informações consolidadas e sumarizadas estejam pré-disponíveis.

O sistema então, com dispositivos sensores associados às rodas dos veículos com smartphones embarcados conectados enviarão as informações a um sistema hospedado em nuvem, em uma topologia clássica de lot - Internet das Coisas, dentro do conceito de Cidades Inteligentes, tal como mostra a figura abaixo (Fig.3).

Figura 3 - Esquema conceitual do sistema
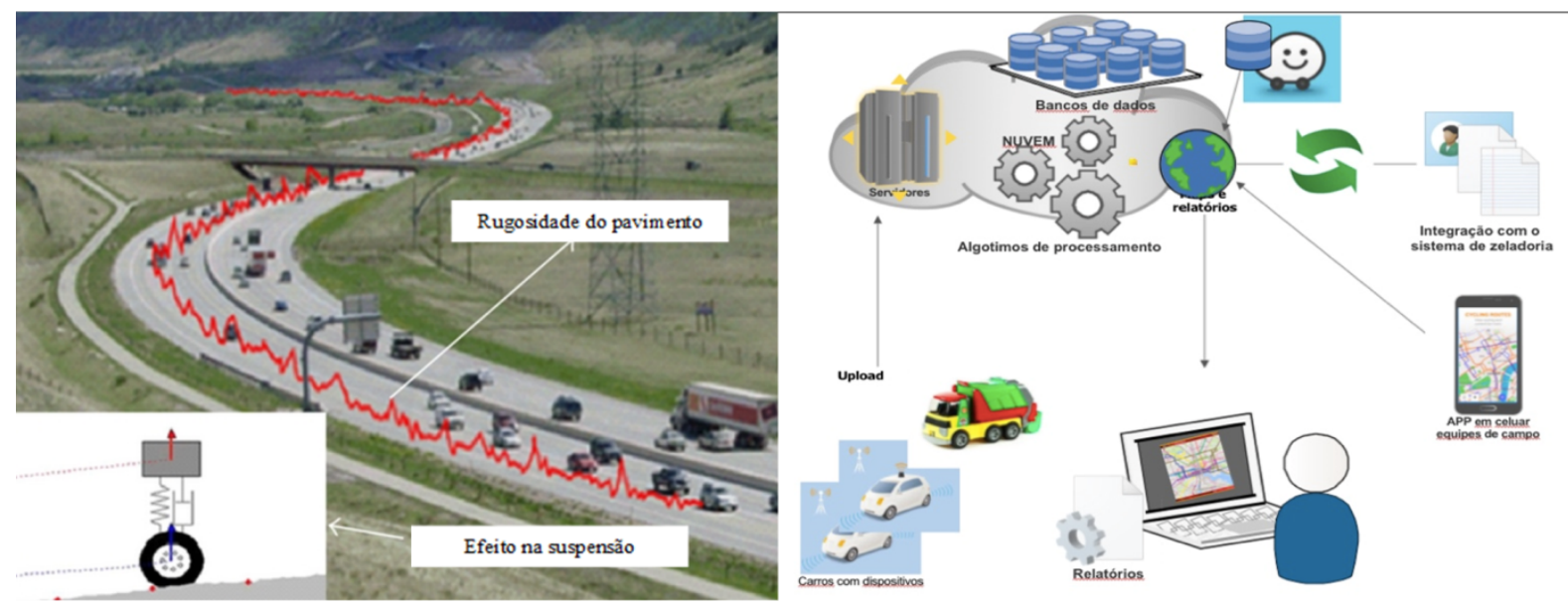

Fonte: Os autores (2019)

\subsection{Instrumentação Hardware dos veículos}

Após o desenvolvimento de uma série de alternativas de testes, o sistema está evoluindo para uma alternativa de sensoriamento através de uma placa de sensores instalada em uma caixa de PVC blindada contra água e poeira, localizada na parte inferior do chassi dos veículos, atuando de maneira integrada à um Smartphone localizado na cabine. Tal alternativa foi escolhida pois reúne uma gama de características que são premissas básicas deste tipo de sistema, como custo baixo, precisão na leitura dos dados, boa captação de antenas GPS, robustez, conectividade com redes de dados, possibilidade de atualização remota de softwares processamento e armazenamento local mínimo de informações.

\section{RESULTADOS}

Após terem alguns veículos com o sistema instalado, a resultante da captação e processamento dos dados tem, atualmente, sido exibida em mapas plotados na ferramenta Mapbox da plataforma Power Bl. De acordo com as respostas de aceleração obtidas, foram definidas legendas de qualidade indicativas em cinco níveis, de acordo com o cálculo do Desvio Padrão das amostras, (ótimo, bom, médio, ruim e péssimo) e plotados no mapa em cores conforme seu nível.

Para aferição e testes dos modelos experimentais foram escolhidos dois trechos, capazes de fornecer informações suficientes para a modelagem do projeto. Foram avaliados nestes trechos, de acordo com a característica dos seus pavimentos, a sensação de conforto do usuário ao trafegar e a repetibilidade dos dados obtidos.

O primeiro trecho de testes escolhido trata-se da Av. Guilherme Villares, na zona sul de São Paulo, que além de possuir um alto volume de tráfego teve durante o processo de monitoramento uma intervenção de recuperação total do pavimento, até suas camadas inferiores de base e sub-base (Fig.4). 
Figura 4 - Apresentação das leituras Geoprocessadas em mapas

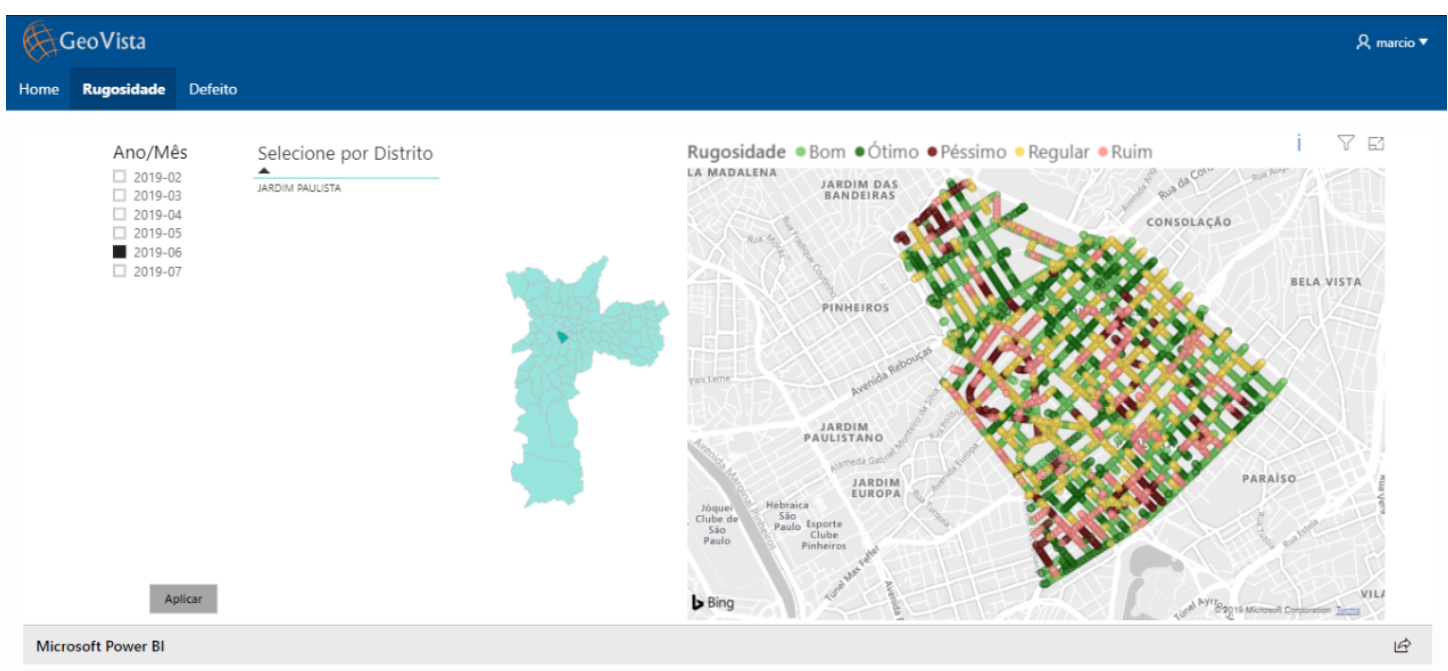

Fonte: Os autores (2019)

Como pode ser notado na Figura 5 abaixo, a plotagem da reação da aceleração dentro da escala definida, foi devidamente alterada correspondendo cada ponto, exatamente ao percebido fisicamente devido à obra de recuperação da via realizada pela prefeitura.

Figura 5 - Resultados Av. Guilherme Villares em diferentes datas

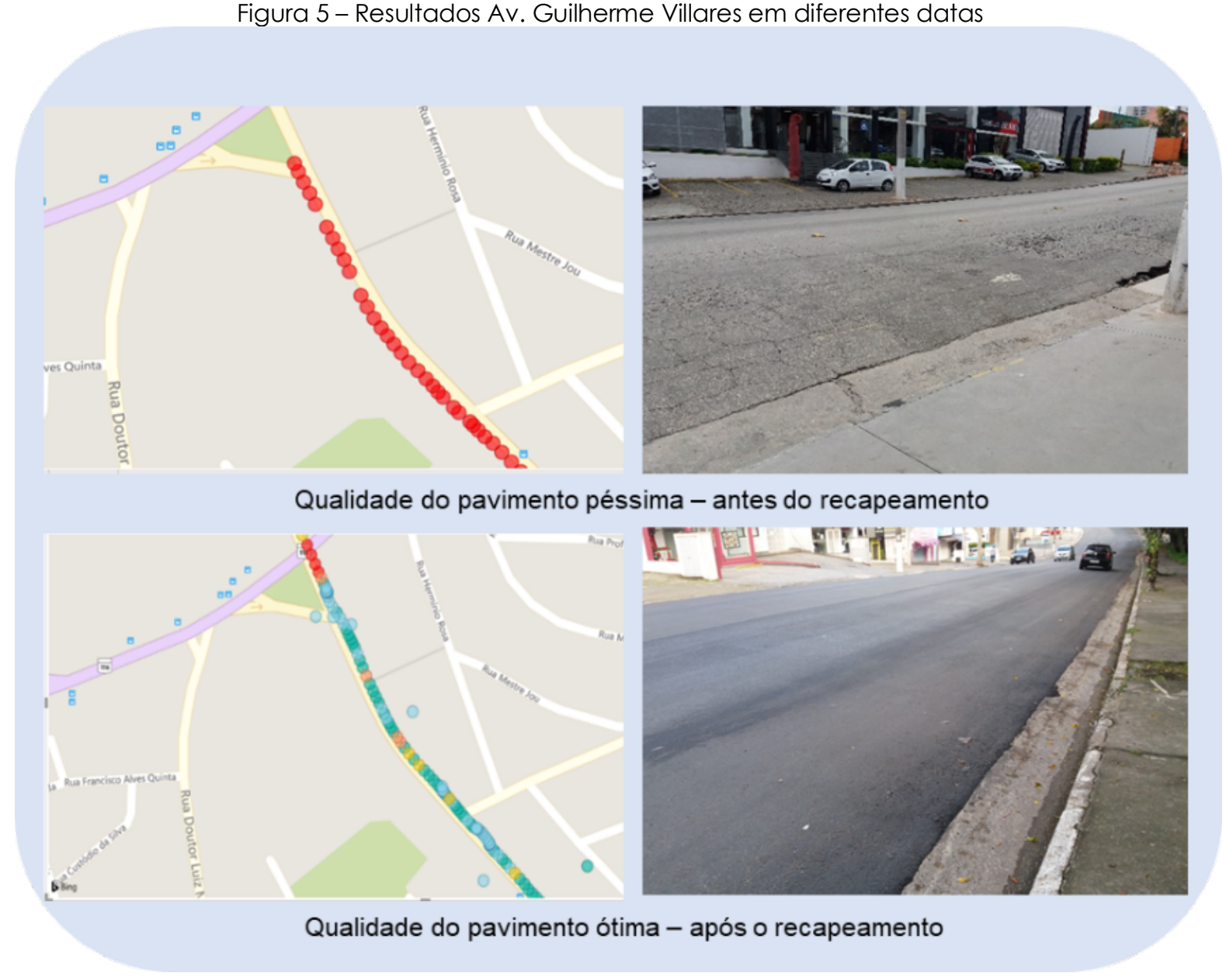

Fonte: Os autores (2019) 
O segundo percurso de testes é um circuito localizado dentro do campus da USP, escolhido por possuir uma gama de pavimentos de diferentes características com diferentes estágios de conservação. Neste percurso foram realizados ensaios com o equipamento MERLIN, que é, dentro da área de pavimentos, um dos métodos consolidado de determinação da rugosidade, podendo assim, determinar o Índice de Irregularidade (IRI) para compará-lo com a solução proposta, calculando a sua correlação:

Figura 6 - Visualização dos resultados de ensaio MERLIN na Volta USP

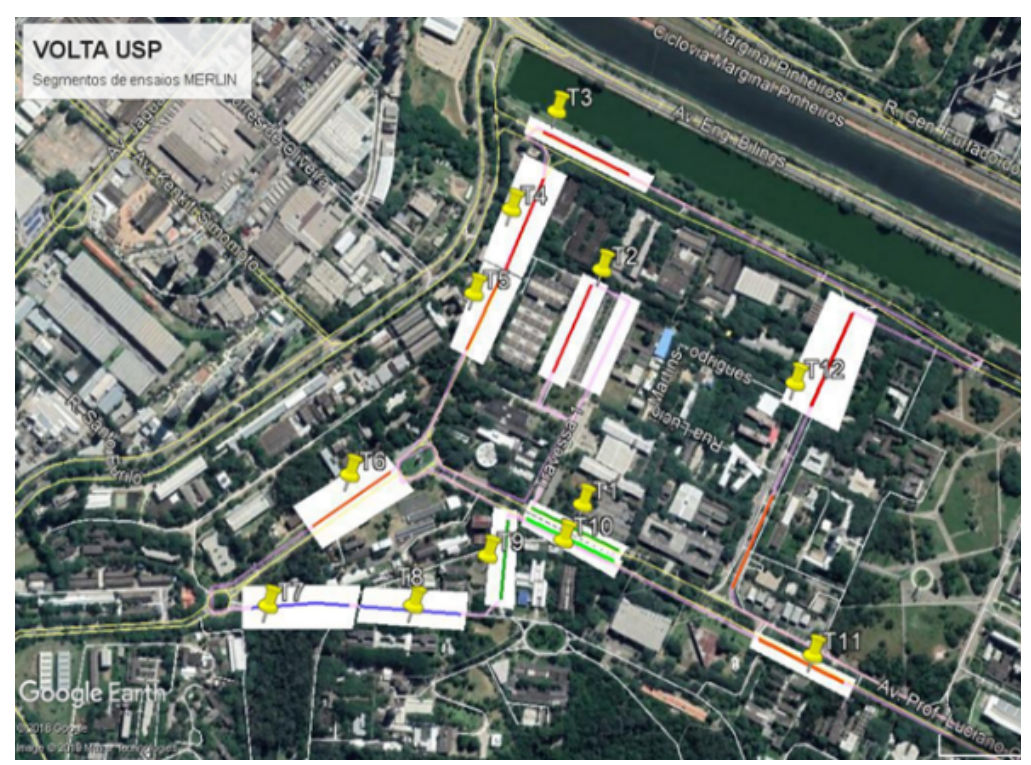

Fonte: Os autores (2019) 
Figura 8 - Correlação dos resultados coletados na Volta USP

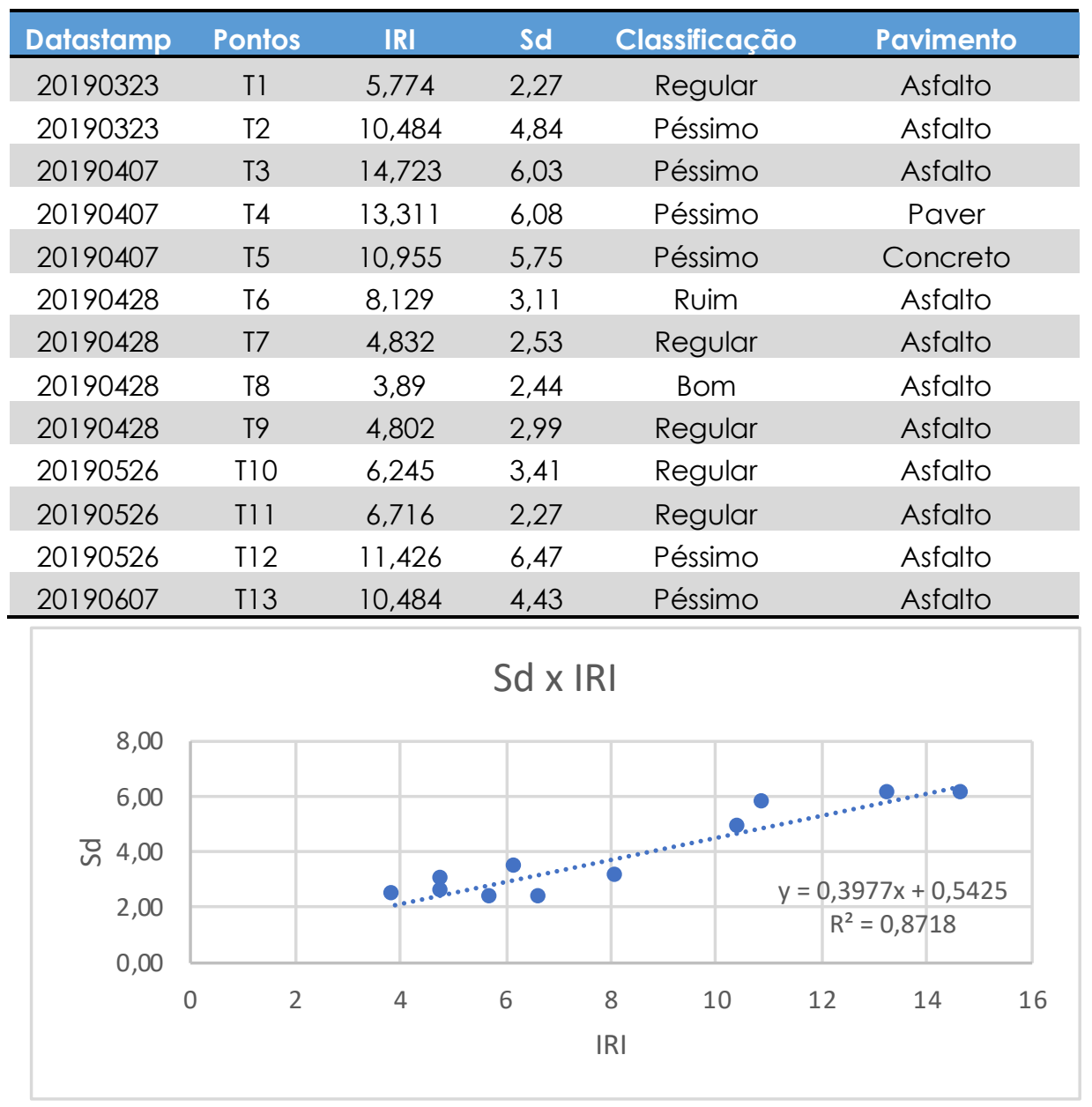

Fonte: Os autores (2019)

\section{CONCLUSÕES}

O sistema em seu estágio atual de desenvolvimento vem apresentando resultados dentro do esperado ao rigor da sua proposta, de avaliar o pavimento de maneira sistêmica e automática através de sistemas de TICs. Interessante ressaltar também a capacidade de expansão do sistema, pois depende apenas da instalação de um hardware de baixo custo numa pequena frota que transite em determinadas cidades, e na devida integração dos dados em escala e com plena utilização por seu público-alvo. Ainda dentro da proposta do estudo, os próximos passos são chegar à fase de comprovação científica definitiva dos resultados obtidos, atualmente, apresentando correlação com nível de confiança maior que $80 \%$, carece ainda de criar modelos estatísticos e matemáticos, com o devido embasamento e comparação com resultados de outros tipos de testes consolidados pela comunidade científica, acadêmica e pelo mercado.

\section{REFERÊNCIAS}

ALESSANDRONI, G.; CARINI, A.O; LATTANZI, E.; FRESCHI, V.; BOGLIOLO, A. A Study on the Influence of Speed on Road Roughness Sensing: The SmartroadSense Case. Sensor (2017). 305

BEUVING, E.; De JONGHE, T.; GOOS, D.; LINDAHL, T.; STAWIARSKI, T. Environmental Impacts and Fuel Efficiency of Road Pavements. European Roads Review. 2. 2004. Disponivel em: http://eapa.org/usr_img/position_paper/fuel_efficiency_report.pdf 
BELZOWSKI, B.; EKSTROM, A. Evaluating Roadway Surface Rating Technologies: Final Report. 2015. 65p. disponível em:

https://www.michigan.gov/documents/mdot/RC1621_493901_7.pdf

BISCONSINI, D. Avaliação da Irrugularidade Superficial dos Pavimentos com dados Coletados por Smartphones. Dissertação (mestrado). Escola de Engenharia de São Carlos da Universidade de São Paulo. 2016.

LIMA, L.; AMORIM, V.; PEREIRA, I.; RIBEIRO, F.; OLIVEIRA, R. Using Crowdsourcing Techniques and Mobile Devices for Asphaltic pavement Quality Recognition. Brazilian Symposium on Computing System Engineering, VI. 2016. P. 144-149

PATERSON, W.; ATTOAH-OKINE, B. Summery Models of Paved Road Deterioration based on HDN-III. Transportation Research, 1344. 1992

PAWAR, Prashant; MATEW, Dr arun; SARAF, Mf. IRI International Roughness Index): An Indicator of Vehicle Response. Materials Today: Proceedings 5 (2018) 11738-11750

TOMIYAMA, K.; KAWAMURA, A.; NAKAJIMA, T.; ISHIDA; JOMOTO, A.. A Mobile Profilmeter for Road Surface Monitoring by Use of Accelerometers. Disponível em:

https://vtechworks.lib.vt.edu/handle/10919/50450

VITTORIO, A.; ROSOLINO, V.; TERESA, I.; VITTORIA, C.M.; VINCENZO, P.G.; FRANCESCO, D.M. Automated sensing System for Monitoring of Road Surface Quality by Mobile Devices. Procedia - Social and Behavioral Sciences. Volume 111, 5 February 2014, Pages 242-251

WATANADA, T. The Highway Design and Maintenance Standards Models: User's Manual for the HDM-III Model (vol.2). John Hopkins University. 1987 